Sharif University of Technology
Scientia Iranica
SCIENTIA
I RAN I CA

\title{
The effects of thickness on magnetic properties of FeCuNbSiB sputtered thin films
}

 \\ and H.R. Madaah Hosseini ${ }^{\mathrm{d}, *}$
}

a. Institute for Nanoscience and Nanotechnology, Sharif University of Technology, Tehran, P.O. Box 1458889694, Iran.

b. Department of Chemistry and NIS, University of Turin, I-10125 Torino, Italy.

c. Istituto Nazionale di Ricerca Metrologica (INRIM), I-10135 Torino, Italy.

d. Department of Materials Science and Engineering, Sharif University of Technology, Tehran, P.O. Box 1458889694, Iran.

Received 8 August 2016; received in revised form 18 January 2017; accepted 6 May 2017

\section{KEYWORDS}

Magnetic properties;

Thin films;

Ion sputtering;

Residual stresses;

Nanoindentation.

\begin{abstract}
Thin films of $\mathrm{Fe}_{73.1} \mathrm{Cu}_{1} \mathrm{Nb}_{3.1} \mathrm{Si}_{14.7} \mathrm{~B}_{8.2}$ alloy with 200, 500, and $800 \mathrm{~nm}$ thicknesses have been deposited by RF sputtering. Their magnetic properties have been characterized using Alternating Gradient Field Magnetometer (AGFM) and Vibrating Sample Magnetometer (VSM). The effects of residual stresses investigated by nanoindentation experiments were conducted on the as-deposited samples. It is observed that the coercivity of as-deposited films is inversely proportional to the thickness in relation with the residual stress induced during sputtering.

(C) 2017 Sharif University of Technology. All rights reserved.
\end{abstract}

\section{Introduction}

Amorphous and nanocrystalline soft magnetic thin films have been studied intensively due to their potential applications in magnetic devices. They have advantages, such as high frequency operation, miniaturization, and high field resolution [1-4]. Among the soft magnetic materials, FeCuNbBSi or so-called Finemet-type alloys are good candidates for such applications due to their excellent magnetic softness. Microstructure and magnetic properties of FeCuNbBSi thin films have been the subject of different studies [513]. Results have shown that amorphous thin films exhibit different behaviour as compared to the rapidly

\footnotetext{
*. Corresponding author. Tel.: +982166165258;

Fax: +98216616520

E-mail addresses: shivaee.ha@gmail.com (H.A. Shivaee); f.celegato@inrim.it (F. Celegato); p.tiberto@inrim.it ( $P$. Tiberto); alberto.castellero@unito.it (A. Castellero); marcello.baricco@unito.it (M.Baricco); madaah@sharif.edu (H.R. Madaah Hosseini)
}

doi: $10.24200 /$ sci. 2017.4429 quenched ribbons. Magnetic properties of thin films can be influenced significantly by internal stresses generated during deposition as well as by surface irregularities [5-7]. Internal stresses coupled with a non-zero magnetostriction of the amorphous films may result in a high magnetic anisotropy. Surface irregularities may also act as pinning centres for domain walls. Both internal stresses and pinning centres in the films can significantly vary with the film thickness [10]. Therefore, the present study aims to investigate the effects of film thickness on mechanical and magnetic properties of the sputtered thin films.

\section{Experimental}

Thin films with different thicknesses (250, 500 and $800 \mathrm{~nm}$ ) were deposited by RF sputtering in argon plasma. Films with different thicknesses were prepared by varying the deposition time while keeping all the deposition parameters almost constant (RF power of $50 \mathrm{~W}$ and residual vacuum $10^{-7} \mathrm{mbar}$ ). An amorphous ribbon with nominal composition of $\mathrm{Fe}_{73.1} \mathrm{Cu}_{1} \mathrm{Nb}_{3.1} \mathrm{Si}_{14.7} \mathrm{~B}_{8.2}$ was used as target. Crystal 
structure in the samples were analysed using a Philips PW3020 X-ray diffractometer with $\mathrm{Cu} \mathrm{K} \alpha$ radiation. The sample surface was characterized by Atomic Force Microscopy using a nanoscope III from digital electronics. Room-temperature hysteresis loops were measured by Alternating Gradient Magnetometer (AGFM) with $H_{\max }=1000$ Oe. Nanoindentation measurements as a suitable probe for mechanical properties were conducted using a Fischerscope HM 2000 Xym fitted with a Vickers indenter. Indentations were performed in a load-control mode up to $1 \mathrm{mN}$ with a loading rate of $0.02 \mathrm{mNs}^{-1}$. Five to ten indentations were made in each sample, and the results presented are the averages of measurements.

\section{Results and discussion}

Chemical composition of the samples is considered the same as that of the target alloy. X-ray diffraction patterns of as-deposited samples, together with that of the free substrate as referenced, are reported in Figure 1. All the samples show a broad halo peak in the range of 40-50 degree, indicating the presence of a fully amorphous structure in as-deposited samples. There are not any peaks in the substrate pattern that could affect the films' patterns.

Figure 2 shows an AFM image of the film having thickness of $250 \mathrm{~nm}$. Apart from some small imperfections, the surface is rather flat. Magnetic Force Microscopy (MFM) was not able to detect any magnetic pattern on the samples surface. This is almost because of magnetic domains in thin films that are rather large and located in the film plane[4].

Room-temperature hysteresis loops of the samples with different thicknesses are reported in Figure 3. They present a typical soft magnetic behavior with the

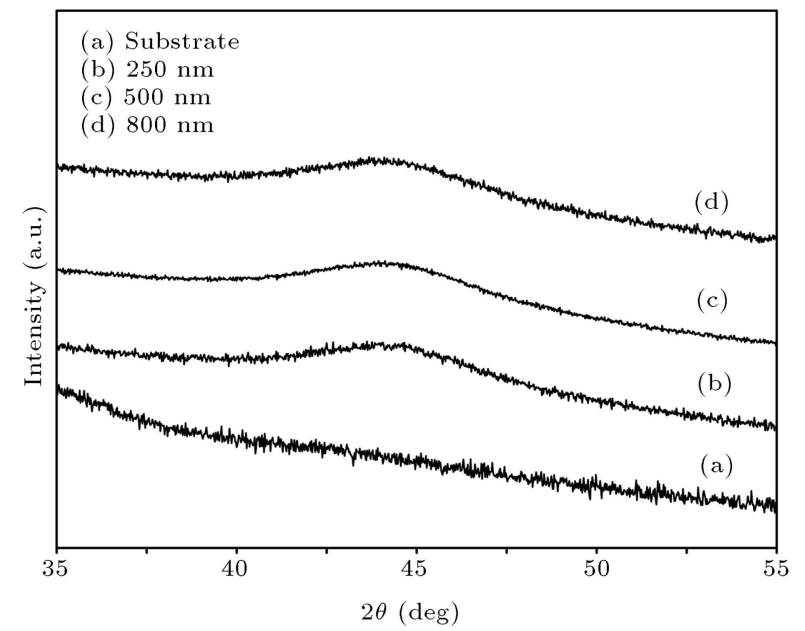

Figure 1. X-ray diffraction patterns of (a) glass substrate and as-prepared films with the thickness of (b) $250 \mathrm{~nm}$, (c) $500 \mathrm{~nm}$, and (d) $800 \mathrm{~nm}$.

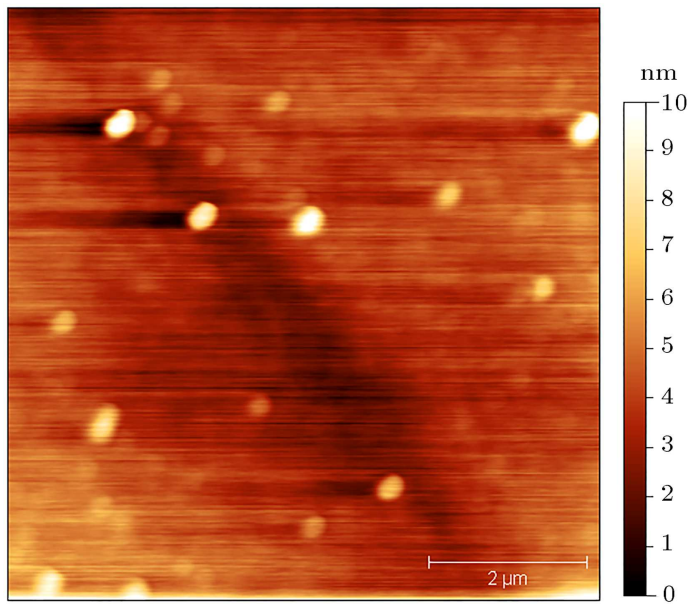

Figure 2. AFM image of the as-deposited film with thickness of $250 \mathrm{~nm}$.

occurrence of a high magnetization jump at low magnetic fields marking high initial magnetic permeability. Coercive field $\left(H_{c}\right)$ values are $40.7,5.6$, and $2.4 \mathrm{~A} / \mathrm{m}$ for 250,500 , and $800 \mathrm{~nm}$ thick samples, respectively. Other researchers [8] report similar results. Relatively high coercive force in the films compared to the ribbon with the same composition and its variation with thickness could be related to the presence of internal stresses in the films and also to the intrinsic bidimensionality in thin films.

It is worth noting that the magneto-crystalline anisotropy, magnetic induced anisotropy, and stress induced anisotropy are the main sources of the coercivity. The induced magnetic anisotropy can be mainly induced by applying magnetic field during the deposition. In the case of nanocrystallization by annealing, when the grain sizes grow up to the critical size (around $40 \mathrm{~nm}$ ), the magneto-crystalline anisotropy affects the coercivity according to random anisotropy model [14]. As the magneto-crystalline



Figure 3. Room temperature hysteresis loops of samples with different thicknesses. 




Figure 4. Typical load-displacement (P-h) curve of the samples.

anisotropy and magnetic-induced anisotropy are not present in this study, measuring the internal stresses can be of help to understand the relatively high coercive force of samples.

Hardness measurement is usually used to characterize residual stress in materials [15]. So, nanoindentation was used to calculate the harness in the films. Figure 4 shows the nanoindentation (P-h) curves of the as-deposited samples. Load-displacement curves in Figure 4 clearly exhibit serrated flow during the indentation, showing a stepped load-displacement curve, which corresponds to the activation of individual shear bands. It is worth noting that the observations of serrations are strongly related to the indentation loading rate. In fact, there is a threshold above which the serrations are not observed anymore. The threshold strain rate was somewhat different for each alloy, suggesting the importance of local atomic arrangements and composition in shear banding [16]. In the case of the alloy studied here, a loading rate of $0.02 \mathrm{mNs}^{-1}$ allows for observing the serrations distinctly.

Figure 5 shows the coercive force together with the hardness of samples as a function of film thickness. Generally, hardness calculations from nanoindentation data are based on Oliver-Pharr method [17]. Error bars on the hardness data reflect the standard deviation calculated from the multiple indentations (5-10 times for each sample). In order to have an accurate hardness measurement on a thin film, a commonly used rule of thumb is to limit the indentation depth to less than $10 \%$ of the film thickness. This approach cannot be used for very thin films. This means that the measurements on the thinner films (250 and $500 \mathrm{~nm}$ ) are affected probably by the substrate. In general, the error, due to the substrate effects, increases with increasing indentation depth and with increasing elastic mismatch between film and substrate. Studies on the indentation of thin films have shown that, for a thin film on a substrate, if the film and substrate have different moduli, the measured contact stiffness will deviate from linearity. The hardness measurement of a soft film is enhanced by the hard substrate, while the reverse is true for a hard film on a soft substrate. The true contact depth is underestimated in the case of a soft film on a hard substrate and overestimated in the case of a hard film on a soft substrate, when compared to the contact depth calculated using the Oliver-Pharr method. Compared to hardness, the nanoindentation measurement of the elastic modulus of thin films is more strongly affected by the substrate. This is because the elastic field under the indenter is not confined to the film itself and extends into the substrate. At very shallow depths, the indentation moduli are equal to the true indentation moduli of the films, but they change quickly with increasing indentation depth and approach the substrate indentation modulus [18,19].

Nanoindentation results on the sample and glass substrate in Figure 5 and Table 1 show similar approaches as mentioned above. However, the difference

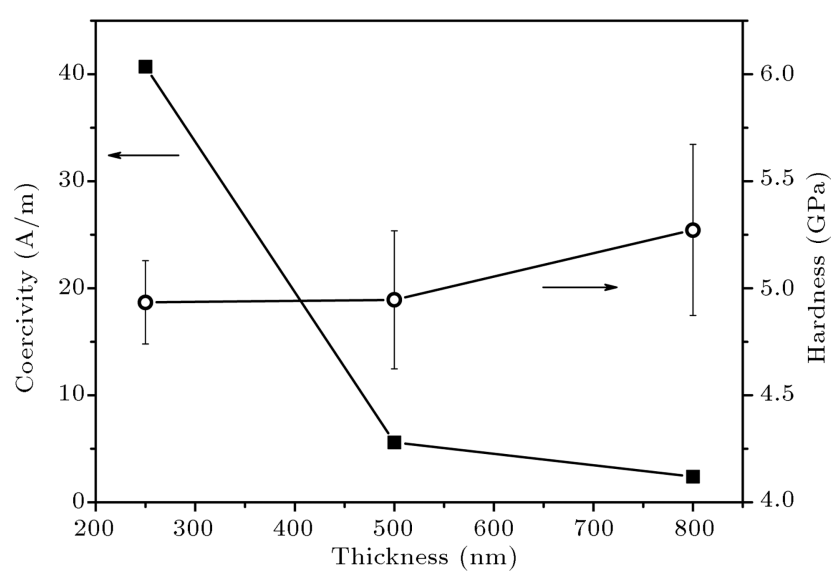

Figure 5. Coercive force and hardness of samples versus the film thickness.

Table 1. Nanoindentation results of samples together with glass substrate.

\begin{tabular}{cccc}
\hline Sample & $\begin{array}{c}\text { Harness } \\
\text { (GPa) }\end{array}$ & $\begin{array}{c}\text { Elastic module } \\
\text { (GPa) }\end{array}$ & $\begin{array}{c}\text { Indentation depth/ } \\
\text { film thickness }\end{array}$ \\
\hline Glass* & $5.1 \pm 0.3$ & $60 \pm 3$ & - \\
$250 \mathrm{~nm}$ & $5.038 \pm 0.2$ & $94 \pm 8$ & 0.3 \\
$500 \mathrm{~nm}$ & $5.051 \pm 0.3$ & $125 \pm 7$ & 0.16 \\
$800 \mathrm{~nm}$ & $5.414 \pm 0.4$ & $119 \pm 6$ & 0.1 \\
\hline
\end{tabular}

* Similar results were reported by other researchers [18]. 
is not so high; hardness and elastic moduli of the films approach the glass values by decreasing the film thickness. Another important issue is that the hardness values obtained for the films deposited on the glass substrate are lower than those measured for the target ribbon. Hardness value measured on the ribbon that was used as a target is $10 \pm 2 \mathrm{GPa}$ in accordance to the literature [20]. This is a possible indication for the presence of residual stress in the films, which is much more significant compared to the small differences observed in the hardness data of thin film samples with different thicknesses. Possible sources for the internal stresses are deposition technique and mismatch between the film and substrate. The sputtering technique is known for inducing high stress in the deposited films, which is inversely proportional to film thickness [5]. It is known that the presence of residual stress affects the overall indentation response of material and, hence, the measured hardness and modulus. Tensile residual stress reduces the hardness, and different levels of residual stress in a film lead to different values of hardness [19]. Therefore, we expected more decrease on the harness of thinner film, but it should be considered that the hardness of thinner films is limited to the substrate hardness. In spite of the hardness, elastic modulus is independent of the residual stresses, including tensile and compressive stresses [21].

Figure 5 also shows that the magnetic coercivity inversely is proportional to the thickness. This can be ascribed to a variety of causes and, mainly, to the internal stresses. Magnetic softness is highly dependent on the internal stress in magnetostrictive materials $[4,5]$. These results suggest that internal stresses increase the coercivity as the thicknesses decrease; these results are in agreement with those reported by some other studies [5-7]. Stresses in the film produced during the sputtering process, acting as pinning centers, give rise to the high coercive field. The combined effect of residual stresses and positive magnetostriction in alloy force the magnetic moments to align toward the stress-induced anisotropy direction. For the thinnest film, the pinning of the domain walls at the surface irregularities might be an excessive reason [5,10]. Increasing the film thickness, the contribution of internal stresses, and surface pinning will become less effective $[5,6]$.

\section{Conclusions}

The magnetic properties of as-deposited Finemet-type $\mathrm{Fe}_{73.1} \mathrm{Cu}_{1} \mathrm{Nb}_{3.1} \mathrm{Si}_{14.7} \mathrm{~B}_{8.2}$ thin films have been correlated to the internal stress. It has been shown that the stress induced by the sputtering is responsible for the high coercive fields of the as-deposited film. It was found that residual stress reduced the hardness. The hardness results of nanoindentation measurements showed that the increase in the thickness leads to the decrease of residual stresses and, consequently, the magnetic coercivity.

\section{Acknowledgment}

H.A. Shivaee is grateful to Univerisità di Torino and Istituto Nazionale di Ricerca Metrologica (INRIM) for the given supports.

\section{References}

1. Mikhalitsyna, E.A., Kataev, V.A., Larrañaga, A., Lepalovskij, V.N. and Turygin, A.P. "Microstructure and magnetic properties of Fe72.5Si14.2B8.7Nb2 Mo1.5Cu1.1thin films", J. Magn. Magn. Mater., 415, pp. 61-65 (2016).

2. Moulin, J., Shahosseini, I., Alves, F. and Mazaleyrat, F. "Ultrasoft Finemet thin films for magnetoimpedance microsensors", J. Micromech. Microeng., 21, pp. 074010-1-07401-8 (2011).

3. Zhang, X., Wang, S., Zhou, J., Li, J., Jiao, D. and Kou, X. "Soft magnetic properties, high frequency characteristics, and thermal stability of co-sputtered FeCoTiN films", J. Alloys Compd., 474, pp. 273-278 (2009).

4. Celegato, F., Coïsson, M., Magni, A., Tiberto, P., Vinai, F., Kane, S.N., Modak, S.S., Gupta, A. and Sharma, P. "Study of magnetic properties and relaxation in amorphous $\mathrm{Fe}_{73.9} \mathrm{Nb}_{3.1} \mathrm{Cu}_{0.9} \mathrm{Si}_{13.2} \mathrm{~B}_{8.9}$ thin films produced by ion beam sputtering", J. Appl. Phys., 102, pp. 043916-1-043916-8 (2007).

5. Moulin, J., Mazaleyrat, F., Mendez, A. and DufourGergam, E. "Internal stress influence on the coercivity of FeCuNbSiB thin films", J. Magn. Magn. Mater., 322, pp. 1275-1278 (2010).

6. Neuweiler, A. and Kronmuller, H. "Magnetization processes in amorphous and nanocrystalline $\mathrm{FeCuNbSiB}$ thin films", J. Magn. Magn. Mater., 177-181, pp. 1269-1270 (1998).

7. Sharma, P. and Gupta, A. "Effect of preparation condition on the soft magnetic properties of FeCuNbSiB thin films", J. Magn. Magn. Mater., 288, pp. 347-353 (2005).

8. Li, X.D., Yuan, W.Z., Zhao, Z.J., Wang, X.Z., Ruan, J.Z. and Yang, X.L. "Nanocrystallization processes and reorientation of the magnetic moments of FeCuNbSiB films", J. Magn. Magn. Mater., 279, pp. 429-434 (2004).

9. Balcerski, J., Brzozowski, R., Wasiak, M., Polanski, K. and Moneta, M. "TEM, XRD and DSC analysis of thin films and foils of FeSiBNb alloys doped with Mn", Vacuum, 83, pp. S182-S185 (2009). 
10. Sharma, P. and Gupta, A. "Ion beam sputtered thin films of Finemet alloy for soft magnetic applications", Nucl. Instr. Meth. Phys. Res. B, 244, pp. 105-109 (2006).

11. Thi Lan, N., Mercone, S., Moulin, J., El Bahoui, A., Faurie, D., Zighem, F., Belmeguenai, M. and Haddadi, H. "Magnetic domain-wall motion study under an electric field in a Finemet ${ }^{\circledR}$ thin film on flexible substrate", J. Magn. Magn. Mater., 373, pp. 259-262 (2015).

12. Kumar, D., Gupta, P. and Gupta, A. "In situ surface magneto-optical Kerr effect (s-MOKE) study of ultrathin soft magnetic FeCuNbSiB alloy films", Mater. Res. Express, 1, p. 046405 (2014).

13. Velicu, I.-L., Kowalczyk, M., Neagu, M., Tiron, V., Chiriac, H. and Ferenc, J. "FINEMET-type thin films deposited by HiPIMS: Influence of growth and annealing conditions on the magnetic behaviour", Mater. Sci. Eng. B, 178, pp. 1329-1333 (2013).

14. Herzer, G. "Modern soft magnets: Amorphous and nanocrystalline materials", Acta Mater., 61, pp. 718734 (2013).

15. Ma, Z.S., Zhou, Y.C., Long, S.G. and Lu, C. "Residual stress effect on hardness and yield strength of Ni thin film", Surf. Coat. Tech., 207, pp. 305-309 (2012).

16. Schuh, C.A. "Nanoindentation studies of materials", Mater. Today, 9, pp. 32-40 (2006).

17. Oliver, W.C. And Pharr, G.M. "An improved technique for determining hardness and elastic modulus using load and displacement sensing indentation experiments", J. Mater. Res., 7, pp. 1564-1583 (1992).

18. Saha, R. and Nix, W.D. "Effects of the substrate on the determination of thin film mechanical properties by nanoindentation", Acta Mater., 50, pp. 23-38 (2002).

19. Li, H. and Vlassak, J.J. "Determining the elastic modulus and hardness of an ultra-thin film on a substrate using nanoindentation", J. Mater. Res., 24(3), pp. 1114-1126 (2009).

20. Um, C.Y., Johnson, F., Simone, M., Barrow, J. and McHenry, M.E. "Effect of crystal fraction on hardness in FINEMET and NANOPERM nanocomposite alloys", J. Appl. Phys., 97, pp. 10F504-10F504-3 (2005).

21. Ling, L., Longy, S., Ma, Z. and Liang, X. "Numerical study on the effects of equi-biaxial residual stress on mechanical properties of nickel film by means of nanoindentation", J. Mater. Sci. Technol, 26, pp. 1001-1005 (2010).

\section{Biographies}

Hossein Asghari Shivaee received his $\mathrm{PhD}$ in Nanoscience and Nanotechnology (Nanomaterials) from Sharif University of Technology in 2010. He is currently an Assistant Professor in Islamic Azad University. His research interest is physical properties of nanomaterials.

Federica Celegato obtained her degree in Materials Science at the University of Turin in 2003. She is currently a Technical Collaborator at INRIM in the Nanoscience and Materials Division. Her currently research activity is focused on vacuum techniques to grow metallic/magnetic thin films and bottom up/top down nanolithography process.

Paola Tiberto obtained her $\mathrm{PhD}$ in Experimental Physics at the Polytechnic of Turin in 1993. She in presently a senior researcher in INRIM in the Nanoscience and Materials Division. Her research activity is focused on the study of magnetization process and on magnetotransport properties for magnetoelectronics in nanoscale magnetic materials.

Alberto Castellero obtained his $\mathrm{PhD}$ in Chemical Science at the University of Turin in 2001. He is currently a researcher at the Chemistry Department of the University of Turin. His research activity is in the field of physical metallurgy, focusing on the development of nanostructures from metastable systems.

Marcello Baricco obtained his PhD in Chemical Science at the University of Turin in 1987. He is currently a Full Professor on Materials Science and Technology in the Chemistry Department of the University of Turin. His research activity is in the field of physical metallurgy, focusing on thermodynamics and kinetics of phase transformations and on materials for energy storage.

Hamid Reza Madaah Hosseini is a Full Professor of Materials Science and Engineering at Sharif University of Technology. He received his $\mathrm{PhD}$ in Advanced Materials from Sharif University of Technology in 2000. He supervised numerous theses and published several papers in the field of Magnetic Materials and Nanomaterials. His research interest is focused on synthesizing materials with therapeutic and imaging applications. 